\title{
A Method for Determining Scale Factor of CFAR Detector Based on BP Neural Networks
}

\author{
Dang Ling, Wang Pingjun, Wang Zhikai \\ Dalian Naval Academy, \\ Dalian 116018, China
}

\begin{abstract}
As applying constant false alarm rate (CFAR) detection algorithms, an important task is to determine its scale factor according to given false alarm probability. When analytic expression of scale factor vs false alarm probability is difficult or impossible to be obtained, simulation is adopted traditionally. But the computation of simulation is very large. A method for determining scale factor of CFAR detector based on BP neural networks is proposed in the paper using powerful ability to approximate any non-linear expression. Studies of examples indicate training times of $B P$ neural networks approximating relation between false alarm probability and scale factor can be largely shorten, after nonlinear transformation of natural logarithm is applied to input of BP neural networks. Studies also indicate method for determining scale factor based on BP neural networks can provide high accuracy.
\end{abstract}

Keywords-CFAR, Scale factor, BP neural networks

\section{INTRODUCTION}

Constant false alarm (CFAR) detection algorithm is a digital signal processing algorithm to provide detection threshold which avoids effect of change of background noise power level. CFAR processors are useful for detecting radar targets in background for which all parameters in the statistical distribution are not known and may be nonstationary. When applying CFAR detection algorithm, an important task is to determine its scale factor according to given false alarm probability. But in many cases, only false alarm probability vs scale factor is available, while analytic expression of scale factor vs false alarm probability is difficult or impossible to be obtained. In this case, simulation is adopted traditionally, but the computation of simulation is very large. For example, assume that the false alarm rate is $10^{-6}$, the simulation times which will have to be carried out is at least $10^{7}$. In this paper, a method for determining scale factor of CFAR detector based on BP neural networks is proposed using powerful ability to approximate any non-linear expression. The relation between scale factor and false alarm probability is approximated by BP neural network, scale factor can be given according to the constant false alarm rate using the trained neural network. Studies of examples indicate training times of BP neural networks approximating relation between false alarm probability and scale factor can be largely shorten, after nonlinear transformation of natural logarithm is applied to input of BP neural networks. Studies also indicate method for determining scale factor based on BP neural networks can provide high accuracy.

\section{BP NEURAL NETWORK INTRODUCTION}

Theory has been proven that three layers BP neural network can approximate any continuous function as long as the hidden node scare is enough [1-3]. A BP neural network structure approximating a single-input single-output continuous function is generally shown in Figure 1. Among them, $F_{X}(x)$ is the input value, $x$ is output value, $W 1_{1}, \ldots W 1_{M}$ are the weights for the hidden layer, $W 2_{1}, \ldots W 2_{M}$ are the weights for the output layer, $M$ is the hidden layer nodes.

\section{BP NEURAL NETWORK USED TO DETERMINE THE SCALE FACTOR}

Although the relation between the scale factor and false alarm rate is generally a single-input single-output function, but the network structure shown in Figure 1 is not applied here. While the modified network structure shown in Figure 2 is applied. The reason is illustrated by the following example.

As non-Gaussian interference CFAR detector has received a great deal of attention, the example first considered is the CFAR detector based on automatic censored algorithm in presence of normalized generalized Pareto distribution reverberation (NGPD) presented in [4]. The relation between false alarm rate and the scale factor is following:

$$
P_{f a}=\left(\begin{array}{c}
N-1 \\
k
\end{array}\right) \prod_{j=1}^{k}\left[\frac{T}{\beta}+\frac{N-j}{k-j+1}\right] \cdot \frac{\beta^{\beta+1}}{(\beta+T)^{\beta+1}}
$$

Where, $P_{f a}$ is the false alarm rate, $T$ is the scale factor $\beta$ is shape parameters of NGPD, $N$ is length of sliding window, $k$ is the number of samples after automatic censored processing. The relation between $T$ and $P_{f a}, T$ and $\ln P_{f a}$ are shown in Figure 3 (a), (b) respectively for $\beta=2$, $N=17, k=14$. It can be seen in Figure 3 (a) that in the neighbor of 0 , a small change of $P_{f a}$ will cause a lot of change of $T$, while between 0.02 and 0.05 , big change of $P_{f a}$ caused a little change of $T$, therefore the relation between $T$ and $P_{f a}$ is very complexly nonlinear, then the number of training required will be very much as neural network approximating the relation. However, It can be seen in Figure 3(b) that the relation between $T$ and $\ln P_{f a}$ is almost linear, then the number of training required will greatly decrease as neural network approximating the relation. The 
fact is true, when the same hidden layer nodes is 50 , the error goals are $10^{-8}$, the training times for each network as shown in Figure 1 are more than 10 million, while the training times for each network as shown in Figure 2 only need 23 times.

Second, we consider the example for the SO (smallest of )-CFAR detector, the relation between the false alarm rate and scale factor is [5]:

$$
P_{f a}=2 \sum_{i=0}^{n-1}\left(\begin{array}{c}
n+i-1 \\
i
\end{array}\right)(2+T)^{-(n+i)}
$$

Where, $n$ is length of the leading and lagging windows respectively. The relation of, $T$ and $P_{f a}, T$ and $\ln P_{f a}$ are shown in the Figure 4 (a), (b) respectively for $n=8$.From Figure 4, we can receive the same conclusion as from Figure 3. In addition, we also considered other CFAR detectors, such as $\mathrm{GO}$ (greatest of )-CFAR, OS (ordered statistics)CFAR, etc., the same conclusion can be received.

\section{ACCURACY ANALYSES}

The true value and output of network are showed in Figure 5 (a), 6 (a) after training (for AC-CFAR-GPD and SO-CFAR detector respectively). It can be seen that the output of network almost coincide with the true value. The errors between the output and true value are showed in Figure 5 (b), 6 (b). The error is defined as the square of the difference. It can be seen that the errors almost lower than $0.3 \times 10-7$. In summary, the accuracy of method for determining the scalel factor based on BP neural network is a very high

\section{CONCLUSION}

As applying constant false alarm rate (CFAR) detection algorithms, an important task is to determine its scale factor according to given false alarm probability. When analytic expression of scale factor vs false alarm probability is difficult or impossible to be obtained, simulation is adopted traditionally. But the computation of simulation is very large. A method for determining scale factor of CFAR detector based on BP neural networks is proposed in the paper using powerful ability to approximate any non-linear expression. Studies of examples indicate training times of BP neural networks approximating relation between false alarm probability and scale factor can be largely shorten, after nonlinear transformation of natural logarithm is applied to input of BP neural networks. Studies also indicate method for determining scale factor based on BP neural networks can provide high accuracy.

\section{REFERENCES}

[1] Wu W, Guo R F, Zheng X L, et al. Deterministic convergence of an online gradient method for BP neural networks[J]. IEEE transactions on neural networks, 2005, 16(3): 533 540.

[2] Jiang Xiaoliang, Li chaoqun. The function approximation methods and Matlab realization based on BP neural networks [J]. Microcomputer \& Its Applications, 2004, 23(1): 52 53.

[3] ZHANG Jing, ,PAN Qi_yong. The Realization and Discussion of a Function's Approximation Based on BP Neural Networks [J]. Journal of Changshu College, 2004, 18(4):34 37.

[4] Xu Jiang-hu Zhang Ming-min Hu Jin-hua. A CFAR Detector Based on Automatic Censored Algorithm in the Presence of Reverberation $[\mathrm{J}]$. Journal of Electronics\&Information Technology, 2007, 29(3): 639 642.

[5] He You, Guan Jian, Peng Yingning. Automatic Radar Detection and CFAR Processing [M]. Beijing: Qinghua university publication, 1999.

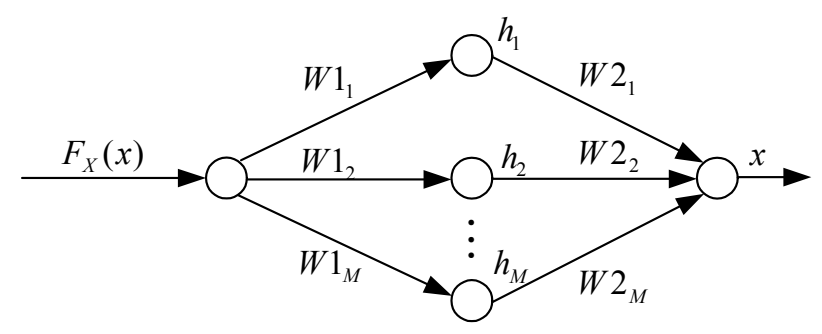

Figure.1 BP neural network structure used to approximate the single-input single-output continuous function

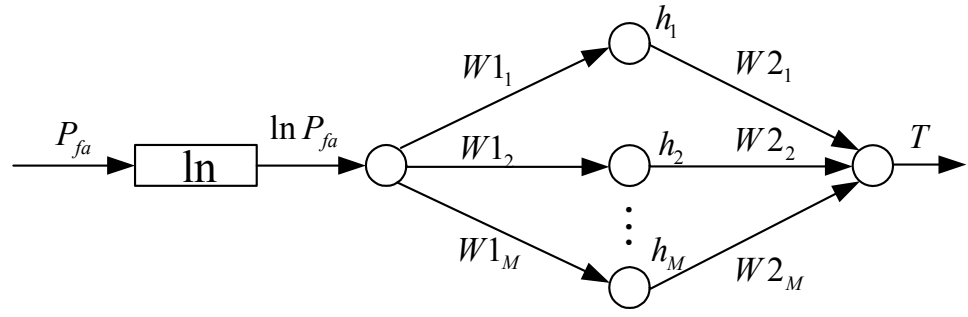

Figure.2 BP neural network used to determine the scale factor 


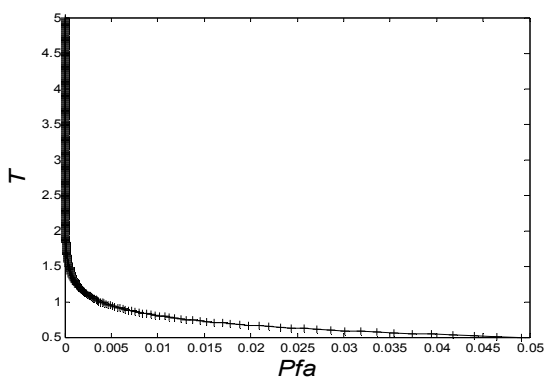

(a)

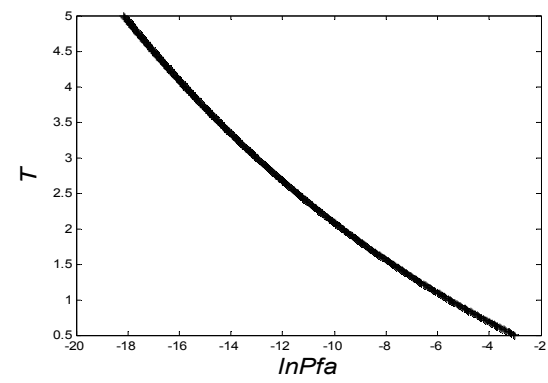

(b)

Figure.3 The relation between $T$, and $P_{f a}$, and $\ln P_{f a}$ for AC-CFAR-GPD

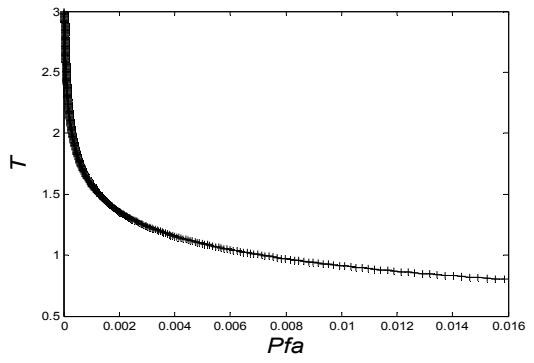

(a)

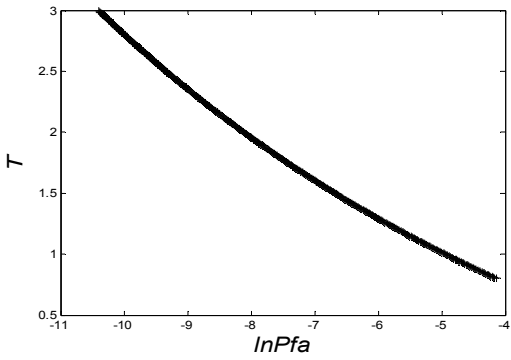

(b)

Figure.4 The relation between $T$ and $P_{f a}, T$ and $\ln P_{f a}$ for SO-CFAR
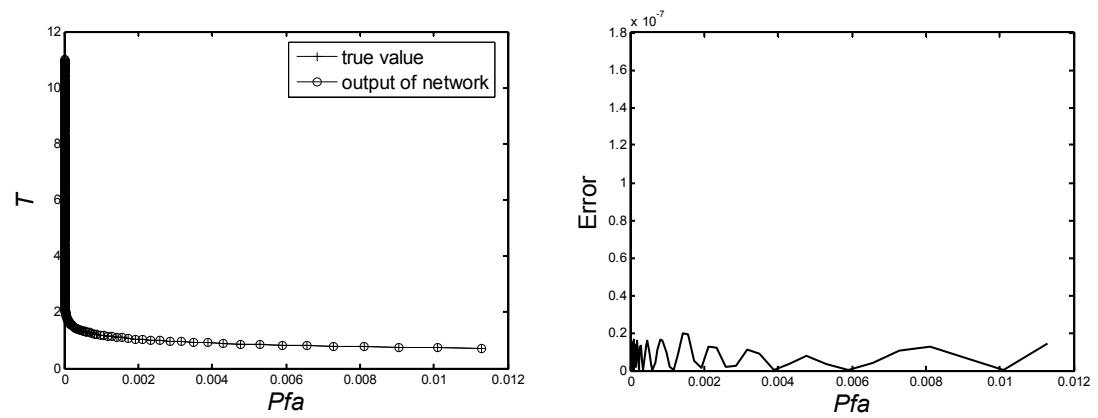

Figure.5 Analysis of accuracy for method for determining the scale factor based on BP neural network (for AC-CFAR-GPD)

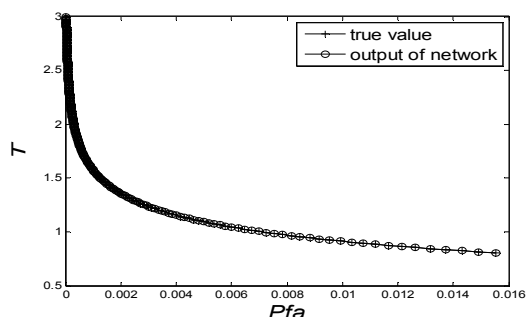

(a)

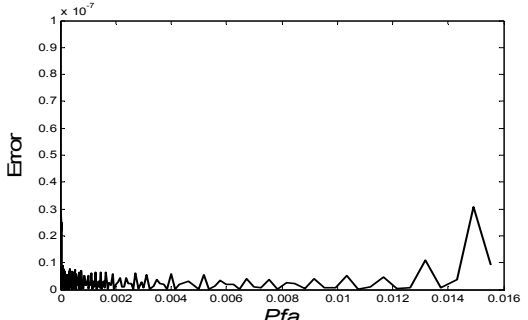

(b)

Figure.6 Analysis of accuracy for method for determining the scale factor based on BP neural network (for the SO-CFAR detector) 